\title{
Malheurs de l'homme et mise à mort rituelle de l'animal domestique dans la société kabiyé
}

The misfortunes of mankind and the ritual killing of domestic animals in Kabiye society

\section{Raymond Verdier}

\section{OpenEdition}

\section{Journals}

Édition électronique

URL : http://journals.openedition.org/span/513

DOI : $10.4000 /$ span. 513

ISSN : 2268-1558

Éditeur

École pratique des hautes études. Sciences humaines

Édition imprimée

Date de publication : 30 mai 1981

Pagination : 155-173

ISSN : 0294-7080

Référence électronique

Raymond Verdier, « Malheurs de l'homme et mise à mort rituelle de l'animal domestique dans la société kabiyé », Systèmes de pensée en Afrique noire [En ligne], 5 | 1981, mis en ligne le 04 juin 2013 , consulté le 21 décembre 2020. URL : http://journals.openedition.org/span/513 ; DOI : https://doi.org/ $10.4000 /$ span. 513 
M A L H E R S DE L 'HOMME E T M I S
A $\quad M O R \quad T$
R I T U E L L E
D E
L ' A N I M A L

$D O M E S T I Q U E$

D A N S

L A

S O C C I E T E

K A B I Y E (1)

par Raymond Verdier

I. Les entités spipituelzes

Monde animal, monde végêtal, monde humain sont animés d'une même énergie issue de la puissance du Dieu créateur eso; cette énergie créatrice kaliza survit à la mort physique et retourne à sa source céleste avant de "sortir" dans un descendant. Ainsi la vie participe de l'oeuvre divine du créateur et la mort est à la

(1). Nous évitons de recourir à la notion de sacrifice pour rendre compte de la pensée religieuse kabiyè; en effet, il ne s'agit pas de "faire du sacré". mais de préserver, par diverses procédures (prières, libations, mises à mort,...) la conjonction des deux faces, visible et invisible, de la vie.

Cette conjonction se trouve mise en échec par la négligence et la faute de 1 'homme, qui, oubliant sa dépendance et subordination vis à vis des esprits protecteurs de la vie, ne respecte pas les interdits et prescriptions de ces derniers. Les esprits entrent alors "en guerre" pour réclamer leur dû, et faute de les apaiser, 1 'home encourt leur malédiction et leur malheur.

C'est dans le contexte de cette disjonction qu'il est question des malheurs et de la misère de l'homme, et de la mise à mort de 1 'animal par laquelle il tente de restaurer 1 'harmonieuse communication entre le visible et l'invisible.

Notre propos n'est pas ici de proposer l'esquisse d'une "thêorie du sacrifice" - ce qui impliquerait une étude comparative délicate et minutieuse -, mais de situer la mise à mort de l'animal domestique dans la pensée et les pratiques religieuses des Kabiyè et d'expliciter le sens qu'ils lui attribuent. 
charnière des deux mondes complémentaires des hommes et des esprits (1).

De même qu'il y a d'un côté les animaux que l'homme élève, de l'autre ceux qu'il chasse (cf. infra), de même les esprits se répartissent en deuxgrandes catégories : il y a d'une part, les esprits protecteurs de 1'individu, de la maison, du champ, du clan, de la cité, d'autre part les génies de la nature sauvage (2).

Ces génies alewa tentent de séduire et d'asservir le voyant, I'homme qui possède la double vue, kinow; entre les alewa qui lui proposent la connaissance médicinale des plantes et le voyant $k i n a-$ du qui leur offre en échange la parole des flûtes, la victoire est au plus rusé, à celui qui réussit à prendre sans donner en contrepartie. Les alewa réclament le sang d'un parent du kinaḍu et l'invitent à leur banquet nocturne; si ce dernier accepte, il devient leur serviteur et sorcier, efelu; si, par contre, il rëussit à leur arracher leur secret contre une flute en beurre de karitë, qui ne tardera pas à fondre au soleil, il en fait ses serviteurs et devient tiw, devin. La communication de l'homme avec les génies sauvages, qui a débuté sous le signe du charme et de la ruse, débouche finalement sur des rapports de force et de domination, qui font, dans un cas, un sorcier, dans 1'autre, un devin : telle est 1a redoutable alternative de ce commerce fatidique pour le bonheur ou le malheur de 1 'homme (3).

A la séduction trompeuse de ces génies errants, qui se nourrissent de la vitalité humaine, s'oppose la surveillance vigilante des esprits gardiens de la vie de 1 'homme et des plantes, garants de la

(1). Cf. notre ouvrage "Citê des Dieux, cité des hommes", Paris, à paraître.

(2). Sur la notion de citê comme entité territoriale socioreligieuse et politique, cf. notre article La cité kabiyè traditionnelle, in Annales de l'Université du Bénin, 1979.

(3). Dans le cas de la sorcellerie, le sorcier mange la vitalité de ses parents pour nourrir ses alewa; dans le cas de la divination, le devin doit sacrifier aux alewa qu'il a domestiqués et qui ne se nourissent plus de chair humaine. 
production et de la reproduction :

- esprits "de sous la terre", aţ̧tuna, ancêtres du foyer et de la maison, qui assurent la continuité de la lignée en "sortant" dans un descendant,

- esprits "de I'eau", timáanyima, qui résident dans les mares et qui en "sortent" pour suivre une femme et devenir les waynyima, "ceux de derrière", de 1 'enfant à nâ̂tre,

- esprits "des champs", hayimnyima, qui font germer la semence et protègent les âmes du mil" contre l'action maléfique des sorciers,

- esprits du clan et de la cité, akolma, médiateurs ultimes entre 1'homme et Dieu, dispensateurs de la pluie, chargés de défendre le territoire contre l'ennemi extérieur et de veiller au respect des usages et à $1^{\prime}$ accomplissement des rituels.

La rêusite de tout ce que l'homme entreprend dépend de leur bon vouloir et il ne peut escompter leur bienveillance que s'il se conforme à leurs prescriptions et interdits. Il leur est en effet redevable de la vie, de la santê et de la prospérité; c'est de la rencontre des deux principes spirituels de l'énergie créatrice kaliza et de la force génératrice, waytu, qu'il procède : la procréation résulte de la conjonction du kaliza et du waytu, issus des waynyima; par ses principes vitaux, l'home se trouve ainsi lié aux esprits et il est leur obligé par une relation nécéssaire et vitale (I).

Aussi l'homme ne peut espérer vivre une vie longue et paisible, disposer d'une nombreuse descendance, avoir de bonne récoltes, s'il ne respecte pas leurs exigences et ne s'assure pas de leur bienveillance. Point de malheur qui ne soit interprêté comme le signe d'une transgression, comme la sanction d'un mal commis. La misère kunygr exprime la souffrance de 1 'homme qui outrepasse sa condition, rompt le dialogue avec les esprits, viole les principes qui ordonnent la

(1). Sur les principes spirituels de la personne, cf. notre article, Contribution à l'étude de la notion d'être humain dans $1 \mathrm{a}$ pensée et la sociêté kabiyè, in La notion de personne en Afrique noire, acte du colloque international du C.N.R.S. 1973. 
vie en société. Elle est la conséquence du malheur qui conduit 1 'homme à la pauvreté kunygntutu, elle est cette "chose qui pousse" (kunyøy de nyøuu, pousser sur) au détriment de la vie, qui l'empêche de crô̂tre et de se multiplier, qui frappe 1 'home de stêrilité, les nouveaux-nés de mort, la terre de sécheresse, le bétail de la peste...

\section{Santé et sainteté : l'élu de Dieu}

La sainteté - tọm wg kaḍ (1) - va de pair avec la santé tonutaa lizin, litt. la douceur de la peau, et la paix-ninka, litt. le froid, la frâ̂cheur; elle manifeste la puissance bénéfique et protectrice de Dieu contre tout kiwgkim, fait ou acte qui rompt et corrompt - wgku - 1'ordonnancement vital des êtres et des choses.

L'êlu et représentant de Dieu, le jQjQ, témoigne de sa saintetê; responsable de la pluie et de la paix sur le territoire de la cité qu'il ne doit pas quitter, il ne peut commettre aucune violence physique ou morale. Sa parole unit et réconcilie, kp̧ntu, elle rêpare et restaure, dahnu, elle redresse ce qui dévie par rapport à la norme, elle ré-approprie ce qui est impropre à l'ordre du monde, kitgkgtim; il ne peut frapper personne, même sa femme, attenter à la vie des plantes, tuer un animal. Son corps est saint, on ne doit pas lui serrer la main quand on le salue, il doit s'asseoir et se coucher sur une peau de chèvre. Exempt de tout mal, il ne doit souffrir d'aucune maladie et doit être en bonne santé. Ainsi protégé,

(1). L'adjectif kadg exprime au sens moral l'idée de mẻchancetê, d'égoìsme; d'un homme avare, on dira qu'il est kaḑ; au sens spirituel, le mot désigne le respect religieux, la pieuse vénêration à l'égard d'une personne, d'un lieu, d'un rituel : ainsi de la prêtrise, on dira : kibalu tom wg kadg, 1 'affaire du grand est sainte; du lieu de fondation du clan, on dira : kgtasi taa tom wg kadg; on dira des pierres. sur lesquelles seuls peuvent s'asseoir les prêtres : $P G$ tom w kadg; les rituels ancestraux sont pareillement qualifiés :

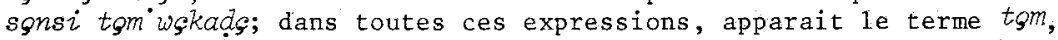
dans le sens diaffaire (au sens premier, le mot désigne la parole). 
Mise à mort rituelle de l'animal domestique dans la société kabiyè 159

par les nombreux interdits de sa charge, du mal, de la maladie et du malheur, il est seul habilité à se rendre dans les lieux saints kgtasi de fondation du clan :

- pour implorer la pluie et demander pardon pour toutes les transgressions qui suscitent le courroux et le châtiment des akolma,

- pour remercier les akglma de donner la santé aux humains et la fécondité à la terre qu'ils cultivent,

- pour oindre de la terre du clan les jeunes enfants lors des fêtes des récoltes de I'igname kiygna en août et du mil sankayin en décembre, ainsi que les initiés kondana (H) et akkpema (F), qui, pour la première fois, pénètrent dans le sanctuaire classique.

Lors des grands rituels agraires et cynégétiques, le jojo se rend dans les lieux saints pour invoquer les akglma et es $Q$ verser l'eau sur terre, ţtu, et le cercle de pierres, kooka, qui figure le lieu de fondation et la puissance du clan; on dit que par ses libations, il "fait entrer la fraicheur (= la paix) dans le pays".

La prière ngh low (1itt. jeter la bouche) peut revêtir des accents pathétiques, oì le prêtre exprime son désarroi, reproche aux akgZma leur négligence et adresse finalement à Dieu ses dolêances :

- Prière à 1'gkglmiyg Kantoh, village Faren, Clan Kumbere ouverture des chasses (janvier 1965)

'Kantoh, ne considère pas les fautes de tes enfants

'Si quelqu'un est contre toi, dresse-toi contre lui !

'Tu dois surveiller strictement la communauté au lieu

'De la négliger et de considérer les fautes de tes

'Enfants. A qui appartiennent-ils si ce n'est à toi !

'Nous ne comprenons pas que tu nous négliges !

'Pourquoi cela ? nous te remercions cependant.

- Prière à 1'gkgtmyg Cacara, village Faren, Clan Cacara

'Dans les années passées, nous quittions ce lieu

'Et la pluie nous accompagnait.

'Aujourd'hui j'ai semé et je ne récolte pas de mil,

'Il sèche dans le champ; vous avez changé ce monde.

- Prière à l'gkglmyg Kanyagade, villlage Faren, Clan Kumbere

'Ma limite s'étend de Sedudè à Pèsem 
'Je ne trouve aucun fruit, je ne comprends rien !

(il s'adresse alors à Dieu :)

'Tu es celui qui a fait descendre 1 'homme,

'Tu es le seul à connaitre le coeur de 1 'homme,

'Autrefois un enfant qui se promenait en brousse

'Trouvait des fruits à manger;

'Maintenant il n'y a plus rien, oû donc chercher refuge !

'Je ne mens pas, je ne vois pas le chemin.

Ayant achevé de parler, le prêtre, dans un geste d'apaisement et de reconnaissance pour les bienfaits escomptés, procède au rite de "verser 1'eau", lim Zisu : il répand sur les pierres du lieu saint un peu d'eau ou de bière de mil, pour que sa parole soit entendue et exaucée. Il faut que la pluie ne tarde pas trop et permette à la semence de germer, mais elle doit également s'arrêter à temps pour que le mil mûrisse et ne pourrisse pas. A Faren, dans le massif Nord, les femmes de la famille du prêtre du clan Cacara préparent en octobre une pâte de mil et y ajoutent du noir de fumée; les enfants sont invités à consommer dans la petite case-sanctuaire de l'gkglmiyg les boulettes de mil noircies que leur présente le prêtre et qui représentent les épis gâtés du mil; par ce geste, le prêtre invite l'esprit à mieux surveiller les cultures. Lors de 1 a récolte du petit mil en juillet, des ignames en août et novembre, du mil en décembre, les prêtres offriront les prémices aux akglma et les chefs de maison aux atgtuna; c'est seulement à partir de ce jour que la nouvelle nourriture sera consommée...

Prières et libations sont ainsi le véhicule du dialogue direct que le jgjg entretient avec l'invisible; il suffit de la médiation de la parole et de 1 'eau adressês aux aķ lma, pour que s'instaure la communication entre Dieu et son représentant. Dieu n'a pas de sanctuaire, il ne réclame pas de victime, il ne reçoit pas le sang de l'animal immolé. Le prêtre est considéré comme un gkolmiyg et il invoque Dieu pour le bien de tous; home de paix, il n'a pas à sacrifier, il doit donner de l'eau à terre; sa mort n'est pas pleurée et son inhumation a lieu la nuit dans une tombe que l'on creuse spécialement pour 1 ui. 


\section{Malédictions, maladies, mort}

Au temps des origines, l'homme ne mourait pas; la mort fit son apparition avec la guerre, la misère avec la désobéissance de 1a femme :

La lutte du fort et du faible

'Quand les hommes furent nombreux, la guerre apparût

'Entre le faible et le fort.

'Le fort alla partager la couche de la femme du faible

'Le faible alla voir un génie et lui dit : "mon ami, lui

'Et ma femme sont couchés ensemble, que dois-je faire ?"

'Le génie lui répondit : "coupe une épine, et mets la

'Dans le poison; la nuit, quand il viendra, pique le."

'Il suivit son conseil et le fort mourut.

Les deux marmites

'Dieu avait créé le monde et y avait mis beaucoup de vivres,

'Les hommes mangeaient et buvaient en paix.

'Dieu leur interdit de toucher à deux marmites;

'La bonne marmite était blanche et contenait le bonheur

'La mauvaise marmite était noire et contenait le malheur.

'Pendant la nuit une femme alla ouvrir la marmite noire;

'Les abeilles commencèrent à piquer les gens

'Et la misère kuny̧̨ se répandit dans le monde entier.

Pour que terre soit fertile, il faut la débarasser de tout ce qui est nuisible à la vie, il faut enfermer la mort, la "renvoyer", sim donu.

En août, lors de la fête des ignames mâles (kiyêna) l'homme du marché, kiyakudu, tue un poussin sur l'autel du marché, un tumulus de pierres, haut de un mètre cinquante, où la mort est enfermée; il embroche le poussin de la bouche à l'anus et le plante au sommet du tas de pierres.

Lors du grand rituel habiyg en novembre (dgntu dans le massif Nord Lamadesi), les voyants et les hommes puissants dëmolissent 1'autel du marché, puis la nuit venue, partent à la recherche du serpent cincin; après l'avoir découvert, ils l'attrapent, le coupent en morceaux - on dit alors qu'on tue la mort, ba ku sim - et le 
mettent dans une marmite neuve avec de la boue et toutes sortes d'animaux venimeux, scorpions, crapauds. Tout le jour, ils danseront autour de la marmite, en montrant, par leurs exploits, qu'ils sont plus forts que la mort; après lui avoir barrée la route en répandant de 1 a cendre à la croisée des chemins, ils iront jeter la marmite, à la tombée de la nuit, à la frontière du territoire ennemi.

La mort guette 1 'homme au dehors - il doit se protéger contre 1 'ennemi extêrieur -, elle le guette aussi au dedans de la cité. II doit d'abord se prémunir contre le risque de maladie et mort par sorcellerie : qu'un sorcier lui ravisse sa force vitale ciycm, il tombe malade, maigrit et meurt si un devin qualifié n'intervient pas à temps pour le délivrer. La cure divinatoire est le seul remède au danger de mort par sorcellerie. Il doit ensuite prévenir le risque de provoquer le courroux des atctuna et akglma, s'il vient à négliger ses devoirs à leur égard et à "tomber dans le piège" (= à transgresser) des interdits. Il "entre alors en dette" - su kimiyg et s'expose à leur malédiction s'il n'acquitte pas son dû en leur sacrifiant.

Dans leur vie invisible, les esprits continuent d'éprouver les désirs et passions des hommes; faute d'être honorés et respectés, ils en éprouvent du ressentiment et peuvent "arracher sa dette"; ils frapperont l'individu ou son groupe dans sa subsistance (sécheresse, invasion de sauterelles...) ou dans son corps : maladies, morts accidentelles sont des symptômes de leur malédiction et de leur malveillance; on dit alors qu'ils "entrent en guerre contre les gens" (1).

Alors que la mort d'une personne âgée est signe de repos pour celui qui a bien vécu - on danse lors de ses funérailles et il devient atģtu -, la mort accidentelle frappe celui qui a mal agi; le noyé, le foudroyé, l'homme tué dans un accident de la route, ne sont pas enterrếs dans le tombeau familial mais sur le lieu même de

(1). Cf. notre étude Justice, pouvoir et vengeance chez les Kabiyè, in La vengeance (ed. R. Verdier), vol. 1, 1980. 
Mise à mort rituelle de l'animal domestique dans 1 a sociétẻ kabiyè 163

1 'accident.

Lèpre, variole, hydropisie, cécité sont le plus souvent considêrées comme des malédictions des akglma : la lêpre et la variole comme le châtiment du mangeur de la vitalité humaine, l'hydropisie comme la sanction du mangeur de la vitalité des plantes, la cécité comme la réponse à l'adultère commis avec la femme d'un frère aîné; on dira alors : q wgkka yg pi wgkkg i: il a "gâté, ça l'a "gâté".

De même que la divination est le seul recours pour démasquer le sorcier et lutter contre la sorcellerie, de même le sacrifice est le seul remède pour conjurer la malveillance des akglma et atgtuna et "refroidir leur coeur". C'est dans ce contexte que prend toute sa force l'expression proverbiale :

Eyu dow kping s'g wahna g kunygn: I'home élève la bête pour guérir sa misère.

\section{La mise à mort}

Si tout homme a le devoir de "sacrifier", tout le monde ne peut exercer 1 a fonction de sacrificateur, qui est liée au statut personnel et parental.

D'abord 1a, femme ne peut mettre à mort; on en donne trois raisons :

- sa force redoutable qui peut devenir meurtrière et se retourner contre elle; on rapporte le cas de femmes, qui, dans 1'ancien temps, Iors du habiyg, avalaient l'enfant dont elles venaient d'accoucher,

- son état d'impureté quand elle a ses règles (elle ne peut alors faire la cuisine),

- sa mobilité, dûe au fait qu'elle est "prêtée à ses maris", qu'elle continue d'appartenir à sa famille en tant que fille et soeur, qu'elle demeure sous la garde de son frère aîné, qu'on ne l'enterre pas chez son mari, mais chez elle.

Pour être sacrificateur, il faut être chef de maison. Le fils marië, qui n'a pas construit sa maison personnelle et qui continue d'habiter avec le père, ne peut sacrifier lui-même; c'est seulement à la mort du père, chef de maison, ou en son absence, que le fils aîné, ou 
le neveu utérin, pourra sacrifier.

Pour "sacrifier" aux algtuna, il faut être chef de la grande maison, deasgsg, celle où se trouve la case-sanctuaire contenant leurs autels; pour "sacrifier" aux ak@lma, il faut être chef de lignage.

1. On ne peut offrir aux esprits protecteurs que le fruit de son labeur, ce qu:on a produit par son travail et grâce à leur bénédiction. Mil et ignames, les deux principales nourritures, sont offertes en prémices lors des fêtes des récoltes; la pâte de mil, d'igname est alors préparée par les femmes et déposée dans des pierres creuses pour les esprits, avant d'être consommée; la bière de mil est alors versée sur 1'autel et consommée par tous les participants.

Seu1 l'animal domestique peut être sacrifié; aucun animal sauvage ne peut être offert aux esprits, hormis certains oiseaux, le lézard kizaku, ainsi que les crabes et grenouilles que 1 'on peut parfois substituer au mouton dans le sacrifice aux waynima et limdanyima. On dit que les animaux sauvages appartiennent aux alewa; le chasseur qui a tué un animal de brousse doit, au retour de la chasse, procéder à un rituel de sêparation et de protection pour se prémunir contre le risque que 1 'animal mort ne se venge.

Tout animal domestique, à l'exception du porc, du chat, du cheval et du canard (ces deux derniers sont d'importation récente) peut être "sacrifiê"; quand au chien, il fait l'objet d'une réglementation particulière, car il est l'animal de l'initiation des adolescents efala et sa consommation est interdite aux fenmes et aux garçons non encore initiés. Sa mise à mort et sa consommation différent dans les deux massifs (1).

Dans le massif sud (Kidjan-Kèwè), l'animal est étouffé par strangulation; on le tue - ba ku - on ne le "sacrifie" pas - ba la (2) -;

(1). Cf. notre article Le canicide chez les Kabiyè, in Systèmes de pensée en Afrique noire, cahier n०1, 1975, pp. 129 à 136 .

(2). Zaw : "sacrifier"; kizow : "sacrifice". 
durant les trois ans de son initiation, l'efalu mange le chien à l'écart de la maison avec ses camarades de classe d'âge; lors des luttes annuelles, il s'enduit le corps de la graisse de chien. A la fin de son initiation, il n'a plus droit de manger le chien.

Dans le massif nord (Lamadessi), le chien est "sacrifié" sur 1'autel du waytu du nouvel initié; à partir de ce jour et jusqu'à la fin de sa vie, il pourra consommer la viande de chien.

La victime doit être celle désirée par l'esprit : son espèce, son sexe, sa couleur doivent répondre à ses exigences. Pour certains sacrifices, la victime est connue d'avance : ainsi le bélier est 1 'animal sacrifié pour 1 'initiation des jeunes filles akpema et des guerriers kgnáana et pour le rituel d'entrée dans la classe d'âge des esakpa; le coq est sacrifié pour l'efazu et le sankayu; lors des grands rituels d'expiation dans les hauts lieux, on immole un jeune taureau attrapê dans un champ.

Pour d'autres "sacrifices", c'est le devin, consultē pour interpréter les présages kawulasi envoyés par l'esprit, qui transmettra ses volontês et prescrira le sexe et la couleur de la victime. S'il n'a pas l'animal adéquat, le chef de maison devra se le procurer par échange ou prêt auprès d'un parent ou ami; s'il ne trouve pas l'animal souhaité, il demandera à l'esprit de patienter en Iui substituant provisoirement la corde qui sert à l'attacher.

2. Toute mise à mort n'est pas "sacrificielle"; ainsi au début des funérailles, les parents du mort tuent au dehors de la maison de nombreuses bêtes en son honneur. Pour qu'il y ait sacrifice, kilaw, il faut que l'animal soit destiné à l'esprit et tué au lieu où il est invoqué, près de son autel, morceau d'assiette, boule de terre, pierre ronde, bâton fourchu, tas de pierre ou arbre (1).

(1). Chasse et guerre se situent hors de l'espace civique et sacrificiel, dans un mode sauvage où la mort est un simple meurtre : on tue les animaux sauvages, on tue le sorcier, on tue l'ennemi; il n'est point là question de sacrifice. Quand quelqu'un a échappé 
Certains "sacrifices" ont lieu à l'intêrieur ou à l'entrée de la maison, d'autres, au bord du chemin, dans les champs, au marché, enfin dans les lieux saints.

Les "sacrifices" domestiques s'adressent aux waynima, limdanyima et atgtuna; chaque individu a, à l'entrée de sa case, un aute1, le naaka, petit morceau d'assiette recouvrant deux petites pierres ou petits pots, pour son waytu; dans la cour principale, une pierre plantée est destinée aux sacrifices pour les timaanyima de la maison; enfin dans la grande maison familiale se trouve la case des atgtuna contenant les pierres et boules de terre, kumola, des ancêtres réputés. Certaines familles ont, en outre, près du vestibule d'entrée, un arbre ou amas de pierres pour l'ekglmiyg protecteur de la maison.

Les champs ont aussi leurs autels. Quand le mil grandit, on pique un oeuf frais et un jeune poussin sur un bâton fourchu planté en terre. La protection des hayimnyima est alors demandée afin que les âmes du mil ne soient pas emportées par quelque sorcier et détournẻes à son profit. Lors de la récolte du mil, on sacrifie sous l'arbre ou le tas de pierre en reconnaissance aux esprits du champ; l'animal est alors consommé sur place avant de rapporter les épis à la maison.

Le marchê, situë à l'emplacement d'un ancien champ, a aussi son tas de pierres flanqué d'un bâton pointu sur lequel on empale le poussin de la mort (cf, 2.5).

Les akglma ont leur autel, pierres et arbres, dans les lieux saints kgtasi; ce sont des bosquets où 1 'on ne doit pas ramasser le bois mort et où, seuls les jgjgna sont habilités à officier pour l'ensemble de la communaté; dans certains lieux saints, deux petites cases-sanctuaires figurent 1 'habitation du premier ancêtre

à un accident (de chasse, de la route...), on lui passe sur le corps une poule, pour que ses plumes lui enlèvent le malheur; on dit qu'on lui "enlève la poussière" pe kpisi eyu; la poule est simplement tuée et n'est pas offerte à un esprit. Lui seul consome sa chair. 
clanique et de sa. femme.

3. Tout "sacrifice" débute par une prière adressée à l'esprit et une $1 i-$ bation de bière de mil sur son autel. Avant de tuer le mouton ou la chèvre, le sacrificateur présente à l'esprit un poussịn ou poulet, lui arrache les plumes du dos et du derrière, qu'il rẻpand sur 1'autel; puis il 1ui incise le cou et répand quelques gouttes de sang sur 1'autel avant de jeter l'animal à terre. L'assistance observe alors un profond silence durant 1 'agonie du volatile, dont elle suit attentivement les ébats jusqu'à ce qu'il s'immobilise. La victime doit mourir sur le dos, les pattes en l'air; elle a alors "salué" et l'esprit l'a "reçue". on brise alors ses pattes, on l'éventre et, après avoir retiré les intestins, le foie et le gésier, on la donne à cuire aux femmes.

Le mouton ou la chèvre a êté attaché à un bois près de l'aute1; le sacrifiant le détache et le prend par la corde avant que le sacrificateur le renverse et le serre entre ses jambes, la tête en avant et le museau fermé avec sa main gauche (1); il prend alors le couteau dans la main droite et lui transperce la gorge. Le sang est recueil1i dans une marmite et n'est pas versé sur 1'autel. L'anima1 exsangue est placé sur un lit de feuilles pour être dépecé : on lui enlève la peau, on découpe les épaules, puis les cuisses; une épaule et une cuisse iront à l'oncle maternel, une cuisse au neveu, une épaule aux parents du sacrifiant; la cervelle sera pour les enfants et le devin appelé en consultation recevra la cage thoracique; enfin la tête, les côtelettes et le dos seront envoyés aux parents de la grande maison.

Foie, poumons, pancréas et reins sont mis de côté avec le sang, pour être consommés après cuisson par les participants; on les désigne

(1). Lorsqu'il s'agit d'un sacrifice de réparation visant à laver $1^{\prime}$ auteur d'un $k i w s k i m$, on passe $1^{\prime}$ animal sur son corps avant de le tuer. 
par le terme aggtģ la. La panse est vidée de son rumen et ce dernier est répandu tout autour de l'autel. Le cou est découpé en petits morceaux ainsi que la panse et on en fait des petites boules de viande ficelées avec des bouts d'intestin; ces boulettes crues, Zozoha, seront envoyées à tous les ainés de la famille.

Entre temps, les femmes ont préparé la pâte de mil, fait cuire le poussin et les aggtgla pour le repas nitgtg (1). L'esprit a déjà reçu la force vitale, cizyam, du poussin, vêhiculée par le sang et répandu sur I'autel. Le sacrificateur lui adresse quelques paroles et dépose sur 1'autel quelques petits morceaux de viande cuite trempés dans la sauce :

'Aujourd'hui je suis venu, je viens pour que tu regardes

'Dans le fond de mon oeil (= que tu entendes ma requête)

'Qu'ai-je à ajouter ? C'est ta part.

Puis il jette un peu de viande près de 1 ui pour "ceux qui şont avec lui"; enfin il lance un morceau par dessus son épaule à 1 'intention de ses ennemis, "ceux du dehors".

Les participants sont alors invités à consommer la pâte de mil et la viande bouiliie; mise en commun et partage soulignent la solidaritê qui unit entre eux les membres de la communauté familiale et le lien de dépendance vis à vis des puissances qui garantissent sa vie et sa survie.

\section{Le lien de dépendance vitale entre le visible et l'invisible}

1. Pour cerner la place et le sens du sacrifice dans la pensée et l'acti-

(1). Le rite qui consiste à enterrer vivant un animal relève non du domaine sacrificiel, où la victime est consommée, en chair ou en esprit, mais du domaine de la sorcellerie; en pratiquant ce rite, on cherche à faire du mal à son prochain. En règle génêrale, 1 'animal sacrifiê est consommé; toutefois dans certains sacrifices aux akolma, (exemple du chien sacrifiê), 1'animal n'est pas consommé, il est mis dans l'arbre où il pourrira. 
Mise à mort rituelle de I'animal domestique dans la société kabiyè 169

vité sociale des Kabiyé, il faut le saisir dans le contexte global du système de communication entre le monde des vivants et celui des vivants invisibles. L'un et l'autre ne sont pas séparés et opposés comme deux entités distinctes et autonomes; il n'y a pas d'un côté la sphère du sacré, de l'autre celle du profane.

Les deux mondes sont conjoints et complémentaires; ils procèdent l'un et l'autre de la conjonction de ciel et terre, de la descente sur terre des premiers hommes devenus les akglma, les ancêtres fondateurs des principaux clans; d'où le lien indivisible entre Dieu, ses envoyés et les hommes. Ainsi vie et mort font couple et les vivants d'au-delà sont les garants et protecteurs de la vie sur terre : point de rupture avec la vie posthume, comme l'attestent les dernières paroles prononcées à la mort d'un vieux :

'Aujourd'hui tu t'en vas et nous n'y pouvons rien

'Salue ceux qui nous ont devancés et dis leur

'Que nous sommes derrière; va en paix !

Point d'âge d'or ni de paradis perdu, point de faute originelle irrêparable qui aurait dissociê le monde des mortels de celui des immortels, creusé un abîme entre l' humain et le divin, coupé le lien ontologique unissant vie et spiritualité.

Il convient dès lors d'écarter pour la société étudiée le modèle Maussien selon lequel le "sacrifice serait un moyen pour le profane de communiquer avec le sacré par l'intermêdiaire d'une chose consacrée et détruite" (la victime).

2. Le monde des esprits est structuré à 1'image du monde des humains; ce dernier comprend d'un côté le commun des mortels, les "hommes sourds" -kututamu - à la parole des alewa, de l'autre les voyants kinadena, qui entrent en rapport avec eux pour le bonheur ou malheur du genre humain. Divination et sorcellerie sont les deux modes inverses de relation des voyants avec les génies sauvages; il les contrastent avec les procédures mises en oeuvre pour communiquer avec les esprits civilisês, gardiens de la maison, des champs, de la cité, qui veillent à la production et à la reproduction de la vie.

Chaque individu est tributaire de ces puissances spirituelles à 
différents niveaux :

- en tant qu'individu particulier, il dépend des Limdanyima vayrizima,

- en tant que membre d'une famille, il dépend des aţ̧tuna,

- en tant qu'agriculteur, il dépend des hayimnyima.

A ces esprits d'en bas, tout homme doit faire des libations et des sacrifices à divers moments de sa vie, à certaines époques de 1 'année et quand ceux-ci en expriment le désir; au chef de sa maison revient alors la charge d'accomplir le rite sacrificiel approprié.

Enfin, en tant que membre d'un clan et d'une cité, tout individu est sous la sauvegarde des esprits d'en haut, les akglma, à qui seuls sacrifient les doyens de lignage et de clan pour le bien commun sous la conduite du jojo qui les invoque et leur adresse des libations.

esprits du monde sauvage, (alewa)

divination +

sorcellerie $\quad+$

kezisa

kizow esprits du monde civilisé

: (atgtuna, akgtma...)

3. Alors que divination et sorcellerie instaurent un rapport de domination et de soumission entre 1 'homne et les alewa, invocation, noh low, libation, kelisa, "sacrifice", kizaw, expriment le lien de dépendance rëciproque et de subordination hiérarchique de 1 'homme vis à vis des esprits sustentateurs et gardiens de la vie(1).

(1). Cette subordination ressort explicitement dans le conte suivant :

'Une femme était stêrile, on l'amena sous l'arbre (gkolmiyg)

' $L$ 'homme qui 1 'accompagnait dit : "ton premier enfant lui sera consacré".

'La femme devint enceinte et accoucha d'une fille; 
Prier, verser l'eau, "sacrifier" sont un devoir impératif : 1 'homme est l'obligé des esprits et ceux-ci ne manquent pas de lui rappeler par des signes prémonitoires son obligation de dialoguer avec eux et de les honorer sous peine de s'exposer à leur malédiction.

Les esprits ont en effet besoin de commercer avec les hommes, ils ont faim et soif en esprit de cette nourriture qu'ils dispensent aux hommes et ils doivent être payés de retour, et pour les bienfaits qu'ils leur procurent, et pour les offenses qu'ils subissent : par la prière, l'homme sollicite leur bénédiction et pardon, par la libation, il leur exprime sa gratitude, par le sacrifice, il acquitte son dî.

En ce sens, le "sacrifice" est 1 'aboutissement du rituel de communication inauguré par la prière; 1 a mise à mort de l'animal, sa "réception", le partage de la victime, le repas en commun unissent et ré-unissent les deux mondes solidaires des esprits et des hommes : cette union, sans cesse menacée par la faute des hommes et la colère des esprits, le rite "sacrificiel" tend à la préserver et la restaurer.

Mais on saisit en même temps que l'efficacité et la finalité du rituei de mise à mort n'ont plus de raison d'être quand la correspondance des deux mondes et leur harmonie sont assurées par celui qui témoigne sur terre de la sainteté de Dieu, le jojo, 1'homme de la prière eć des libations, celui à qui toute violence est interdite, celui qui ne peut tuer et qui est néanmoins au centre de l'édifice cu1tue1.

\footnotetext{
'La fillette grandit, elle eut un amant et lui déclara :

'Mon père a amené ma mère sous l'arbre, on doit me tuer

'Alors son amant protesta : est-il permis de tuer un être humain?

'Si I'on doit t'amener sous 1 'arbre pour te tuer, je vais aller

'Chercher un chien, de la farine et des poules.

'Au jour dit, le jeune homme grimpa sur 1'arbre;

'Au moment où ses parents la saisirent pour la tuer,

'Il sauta de l'arbre et déclara : on va sous l'arbre

'Pour trouver la santé, non pour tuer un être humain.

'Voici des animaux, sacrifiez la poule, et si tout va bien

'Tuez le chien. Depuis ce jour, on ne sacrifie plus des êtres 
4. Que1 rapportya-t-il finalement entre le kelisa, la libation et le kizow, cet acte de violence qui tient une place si importante dans la pratique religieuse, alors que Dieu ne réclame pas de sacrifice?

a) Au plan spirituel

La mise à mort de l'animal domestique se situe à l'articulation de la mort et de la vie, en ce lieu paradoxal où un acte de mort débouche sur la vie, où un acte destructeur la préserve et la restaure.

La vie est un bien donné et reçu, qui a sa source en Dieu et qui se transmet par le canal des esprits protecteurs, esprits ancestraux, esprits de l'eau, esprits de la terre cultivée... Ceux-ci ont le pouvoir de donner la vie et de la reprendre : ils peuvent bénir et maudire. De leur bénédiction dépend le bonheur de 1 'homme et sa richesse; leur malédiction cause son malheur et sa misère.

C'est dans le contexte de cette dépendance vitale qu'ont lieu libations et offrandes de prémices d'une part, mise à mort de 1'animal domestique d'autre part.

b) Au plan social et politique

La mise à mort rituelle de l'animal domestique débouche normalement sur sa consommation et en principe sur le repas conyivial; pratiques essentiellement communautaires, kelisa et kizow définissent des unités comnensales dépassant le cadre du foyer, rassemblant tantôt les gens de la maison ou de la grande maison patriarcale, tantôt les consanguins et utêrins, dans les grandes occasions enfin, les représentants des clans de la cité. C'est dire que le "sacrifice" circonscrit en dernier ressort l'espace interne de la cité, celui où I'on prend habituellement sa première épouse, celui de tous les hommes qui participent au culte des grands esprits protecteurs akolma; espace qui s'oppose à l'espace du dehors, lieu de la chasse et de la guerre, lieu oũ la mort est un meurtre qui appelle vengeance.

espace du dedans de la citề
kelisa-kizaw
mariage


c) Au plan éthique

La dẻpendance à l'égard des esprits protecteurs s'exprime à deux niveaux : santé et prospérité vont de pari avec ce qui est bon kidgdim -, ce qui est propre - cricri; la maladie - kutgn - et la misère - kunygy-vont de pair avec la transgression $k i$ tg $k g$ tim et la corruption kiwgkim.

Dans le premier cas, l'homne, récompensé de sa bonne conduite, a un devoir de reconnaissance, une dette de gratitude envers les esprits qui l'ont protégé; dans le deuxième cas, celui qui a offensé les esprits et encourt leur malédiction a le devoir d'obtenir leur pardon, de "réparer"; il a une dette d'offense kimiyg, qu'il doit acquitter à temps sous peine de se voir "arracher sa dette", d'être châtié sans appe1.

A ces deux obligations, devoir de gratitude et dette d'offense, se trouvent correspondre les deux rituels du kelisa et du kizaw, les deux fonctions de lisiyg et de layu :

Système d'obligation et de prestations

$\begin{array}{lcc}\text { obligations } & \text { devoir de gratitude } & \text { dette d'offense } \\ \text { prestations } & \text { kezisa } & \text { kizow } \\ \text { agent } & \text { zisiyu, jojo } & \text { zayu }\end{array}$

Cette mise en correspondance schématique fait ressortir d'une part la proximité des deux rituels - qui répondent l'un et l'autre à un dû et qui dans la pratique marchent souvent de pair; d'autre part, la distance théorique qui sépare le devoir pieux de l'obligé de la dette impie de 1 'offenseur.

La différence des deux types d'obligation et de prestation - la corrélation du kelisa et du devoir de gratitude, du kiloow et de la dette d'offense - ne saurait signifier que toute mise à mort rituelle est en rapport direct avec la transgression d'une norme et opère une réconciliation et une réparation. Elle nous suggère seulement que le "sacrifice" a un lien privilégié avec la transgression et qu'en tant que réponse aux malheurs de l'homme qui rompt son lien de dépendance à l'êgard de ses tuteurs spirituels et subvertit 1 'ordre du monde, il est, dans son prototype, un mode de rétablissement de la communication, interrompue par sa faute, et de restauration de l'ordre perturbé. 